Research Article

\title{
An Ensemble of Adaptive Surrogate Models Based on Local Error Expectations
}

\author{
Huanwei Xu $(\mathbb{D}$, Xin Zhang, Hao Li, and Ge Xiang \\ School of Mechanical and Electrical Engineering, University of Electronic Science and Technology of China, \\ Chengdu 611731, China \\ Correspondence should be addressed to Huanwei Xu; zhangxin96428@163.com
}

Received 1 September 2020; Revised 5 January 2021; Accepted 28 January 2021; Published 10 February 2021

Academic Editor: Guoqiang Wang

Copyright (C) 2021 Huanwei Xu et al. This is an open access article distributed under the Creative Commons Attribution License, which permits unrestricted use, distribution, and reproduction in any medium, provided the original work is properly cited.

An ensemble of surrogate models with high robustness and accuracy can effectively avoid the difficult choice of surrogate model. However, most of the existing ensembles of surrogate models are constructed with static sampling methods. In this paper, we propose an ensemble of adaptive surrogate models by applying adaptive sampling strategy based on expected local errors. In the proposed method, local error expectations of the surrogate models are calculated. Then according to local error expectations, the new sample points are added within the dominating radius of the samples. Constructed by the RBF and Kriging models, the ensemble of adaptive surrogate models is proposed by combining the adaptive sampling strategy. The benchmark test functions and an application problem that deals with driving arm base of palletizing robot show that the proposed method can effectively improve the global and local prediction accuracy of the surrogate model.

\section{Introduction}

In the engineering design problem, computer simulation is usually applied to replace the real physics experiments. For complex engineering problems, sometimes the performance function is implicit, or due to cost and time limit, the surrogate model is often applied to approximate the real physical model. Commonly used surrogate models mainly include Kriging [1], artificial neural network [2], radial basis function (RBF) [3], support vector regression(SVR) [4], and polynomial response surface(PRS) [5].

When surrogate model is applied, how to find a suitable surrogate model is a difficult task. In order to improve the adaptability of the surrogate model, a reasonable choice is to use a linear weighted combination of different surrogate models, that is, an ensemble of surrogate models. Compared with the single surrogate model, an ensemble of surrogate models can save a lot of time wasted in screening the surrogate models. Many scholars have conducted in-depth research on it and have obtained many good achievements. Huang [6] found that the ensemble of surrogate models has higher prediction accuracy than the single surrogate model.
Yan [7] proposed a new weight function construction method, which has the same accuracy as the optimal submodel and can improve the approximation of the true response distribution. $\mathrm{Lu}$ [8] found that the multisurrogate model has better optimization results than the single surrogate model's. Pan [9] applied the ensemble of surrogate models to the lightweight design of the car body, and the results achieved a better optimization effect. Liu [10] established the ensemble of surrogate models to solve the structure optimization of car parts. Xing [11] assigned weights to three single surrogate models by using the adaptive metropolis-Markov chain Monte Carlo method. Yin [12] compared the application of a single surrogate model and an ensemble of surrogate models in groundwater restoration design optimization problems, and the results showed that the ensemble of surrogate models is more robust. Li [13] proposed a surrogate-assisted particle swarm algorithm, which can effectively balance the global search and local search. Donncha [14] successfully used the ensemble of surrogate models to improve the forecasting system with significant effects. Ouyang [15] used the analysis of variance method to determine the weights of ensemble of 
surrogate models. The comparison results show that the proposed method can not only improve the prediction performance of surrogate model, but also obtain a reliable solution. Chen [16] presented a new ensemble model which combines the advantages of global and local measures. The results show that the proposed ensemble model has satisfactory robustness and accuracy. Zhang [17] proposed a unified ensemble of surrogates with global and local measures for global metamodeling. It is concluded that the proposed model has superior accuracy while keeping comparable robustness and efficiency.

Although some progress has been made in the research of the ensemble of surrogate models, most of the current methods for constructing the ensemble of surrogate models are stationary sampling. The problem with stationary sampling is that, in order to obtain an ensemble of surrogate models that meets the accuracy requirements, the sample size must be large enough. Adaptive sampling can obtain new samples that benefit the quality of the surrogate model, which can minimize the total sample size. However, the current adaptive sampling is often applied for a single surrogate model [18-21]. Only a few scholars combine the adaptive sampling strategy with the ensemble of surrogate models [22, 23]. The remainder of this paper is organized as follows. Section 2 briefly reviews the main steps to establish the ensemble of surrogate models. In Section 3, the ensemble of surrogate models using adaptive sampling strategy based on local error expectations is described. The proposed method is verified by numerical examples and compared with the three classical ensembles of surrogate models in Section 4. Section 5 applies the proposed method to the engineering design problem of driving arm base of palletizing robot. Finally, the conclusions are given.

\section{Establishment of the Ensemble of Surrogate Models}

There are three main steps to establish the ensemble of surrogate models:

(1) Design of experiment: the experiment design methods are applied to determine the spatial distribution of sample points. Experiment design methods mainly include Central Composite Designs (CCDs) [24], Orthogonal Design [25], and Latin Hypercube Design (LHD) [26]. LHD is the most popular sampling method due to good spatial uniformity. The experiment design method used in this paper is also LHD.

(2) Establishment of the ensemble of surrogate models: the surrogate models can be divided into two categories. One is interpolation methods, such as RBF and Kriging. For these methods, the prediction errors of the sample points are zeroes, which has good unbiasedness. The other is the noninterpolation methods, such as PRS and SVR. The noninterpolation methods have certain fitting capabilities, but the surrogate models do not go through all sample points. Therefore, enough sample points are needed to ensure the high accuracy of the surrogate models, which has extremely high uncertainty. In view of the advantages and disadvantages of different surrogate models, the most commonly used surrogate models are the RBF model and the Kriging model. In this paper, these two surrogate models are combined to establish the ensemble of surrogate models. The expression of the ensemble of surrogate models is as follows [27]:

$$
\hat{y}_{e}(x)=\sum_{i=1}^{N} \omega_{i} \hat{y}_{i}(x), \sum_{i=1}^{N} \omega_{i}=1 .
$$

where $\hat{y}_{e}$ is the predicted response value of the ensemble of surrogate models and $N$ is the number of surrogate models. $\omega_{i}$ is the $i$ th weight coefficient. $\hat{y}_{i}$ is the predicted response value of the $i$ th surrogate model. Generally speaking, the higher the prediction accuracy, the larger the weight coefficient of the corresponding surrogate model.

(3) Accuracy verification: accuracy verification of surrogate model mainly includes two aspects: global accuracy and local accuracy. root mean square error (RMSE) [28] and coefficient of determination $\left(R^{2}\right)$ [29] are two main global accuracy evaluation methods. The corresponding expressions are as follows:

$$
\begin{aligned}
\text { RMSE } & =\sqrt{\frac{1}{n} \sum_{i=1}^{n}\left(y_{i}-\hat{y}_{i}\right)^{2},} \\
R^{2} & =1-\frac{\sum_{i=1}^{n}\left(y_{i}-\hat{y}_{i}\right)^{2}}{\sum_{i=1}^{n}\left(y_{i}-\bar{y}\right)^{2}},
\end{aligned}
$$

where $y_{i}$ is the actual response value of the $i$ th test sample and $\hat{y}_{i}$ is the predicted response value of the surrogate model of the $i$ th test sample. $\bar{y}$ is the mean value of the actual response value, and $n$ is the size of test sample points. For RMSE, the smaller the value, the higher the global prediction accuracy. The range of $R^{2}$ is not greater than 1 . The value of $R^{2}$ can be negative if the fitting quality of the surrogate model is extremely low. The closer the value of $R^{2}$ to 1 , the higher the accuracy of the global approximation of the surrogate model. Although RMSE can evaluate the prediction accuracy of the surrogate model, the magnitude of the specific problem greatly affects the value of RMSE, which is not as intuitive and easy to understand as $R^{2}$. The global accuracy evaluation method applied in this paper is the coefficient of determination $R^{2}$.

The local prediction accuracy evaluation method is maximum absolute error (MAE). The expression of MAE is as follows:

$$
\text { MAE }=\max \left|y_{i}-\hat{y}_{i}\right| .
$$

Similar to RMSE, the smaller the MAE, the higher the local prediction accuracy of the surrogate model. In this 
paper, MAE is also used to evaluate the local prediction accuracy of the surrogate model.

\section{The Ensemble of Adaptive Surrogate Models Based on Local Error Expectations}

The existing adaptive sampling strategy of sample points is mainly for a specific surrogate model, which has poor versatility. In addition, due to the inconsistency of the existing adaptive sampling strategies, it will be very complicated to combine the ensemble of surrogate models with the adaptive sampling strategy. In this section, a universal adaptive sampling strategy based on local errors is proposed. By combining the new adaptive sampling strategy, the method to construct the ensemble of surrogate models is proposed.

\subsection{Adaptive Sampling Based on Local Error Expectations.} Since Kriging and RBF models usually can provide good accuracy for fitting highly nonlinear behaviors, so these two surrogate models are used in general engineering problems. At present, the most commonly used adaptive sampling method is the maximin distance approach proposed by Johnson [30]. Jin and Chen [31] made corresponding improvements and proposed the Maximin Scaled Distance Approach. In this paper, we also propose a universal adaptive sampling strategy based on the local error expectations named LEE strategy for different surrogate models and it is proposed to serve the construction of the ensemble of adaptive surrogate models. The process is shown in Figure 1.

The following are main steps of the LEE strategy:

(1) Build an initial surrogate model. First, LHD is used to obtain the initial sample points and obtain their response values. Since high accuracy is not required at the beginning of sampling, for different dimensional surrogate models, the initial number of sample points can be $5 n_{d}, 10 n_{d}$, and $20 n_{d}\left(n_{d}\right.$ is the number of design variables).

(2) Calculate the expected value $E[\mathrm{AE}]$ of the local error. Use the existing sample points and their response values to construct a surrogate model, and use crossvalidation error method (LOO-leave one method) to obtain the local error of each point. The local error of $i$ th sample point is evaluated by the absolute error $\mathrm{AE}_{i}=\left|\widehat{y}_{i}-y_{i}\right|$. Then the local error expectation $E$ $\mathrm{AE}$ ] can be obtained by the following expression:

$$
E[\mathrm{AE}]=\frac{\sum_{i=1}^{n} \mathrm{AE}_{i}}{n}
$$

By using cross-validation error method, each sample point serves as a test point, and the other sample points serve as the sample points that constitute the surrogate model. When each sample point serves as the test point, it can reflect its importance for modeling and the uncertainty around the sample point's location. The absolute error $\mathrm{AE}_{i}$ can reflect

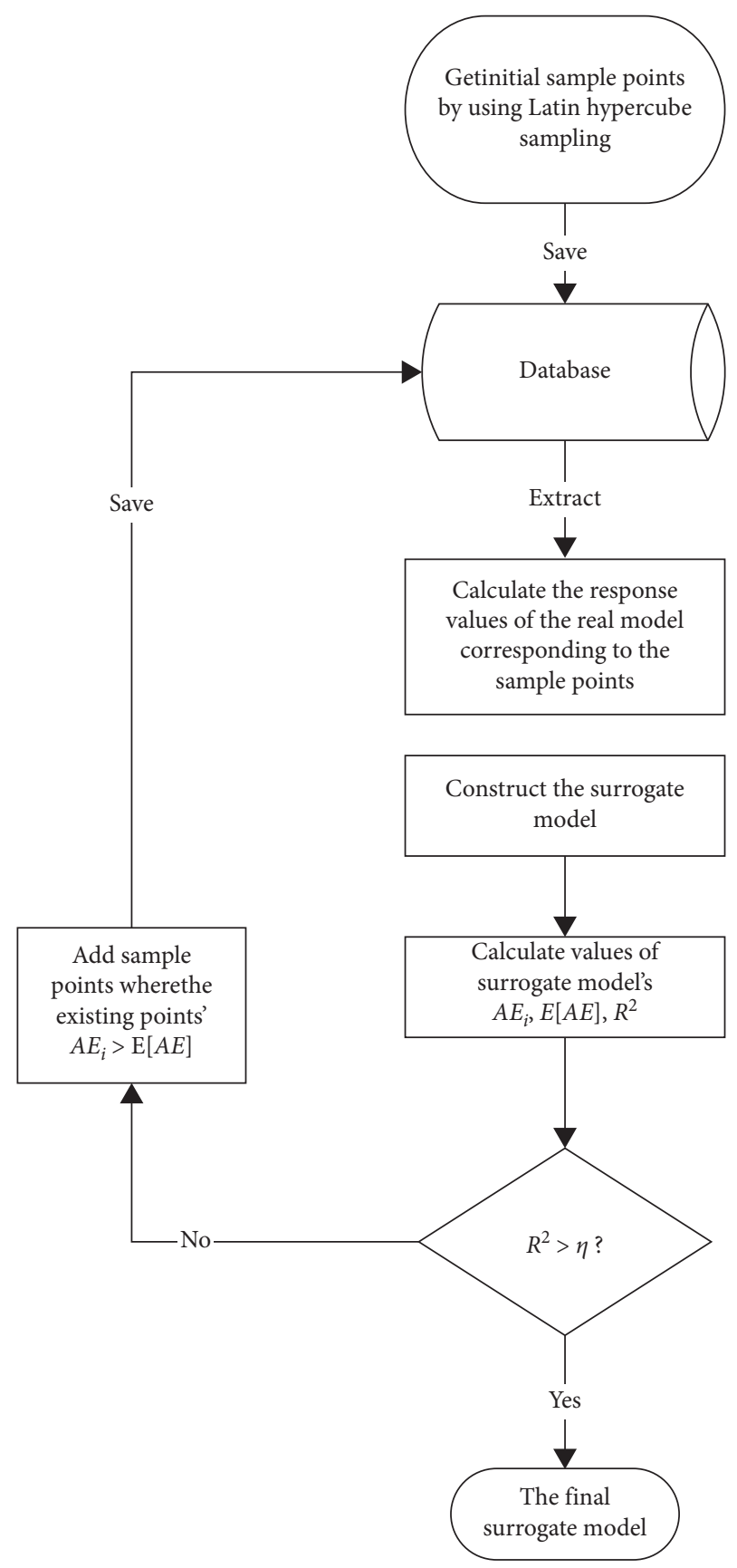

FIGURE 1: The adaptive sampling process based on local error expectations.

the uncertainty around this location, and the expected absolute error $E[\mathrm{AE}]$ of all sample points can reflect the uncertainty of the overall sample points.

(3) Calculate the dominating radius of the sample points. Since the initial sample points determined by LHD have certain uniformity, the same radius can be set for each sample point. $n$ sample points can divide the design space into $n-1$ part. In order to ensure that the radius of each sample point does not intersect as much as possible, we propose the concept of the dominating radius of the sample point. $R_{j}$ is the dominating radius of the $j$ th dimension 
coordinate of the sample point; the expression is as follows:

$$
R_{j}=\frac{\left|x_{j \max }-x_{j \min }\right|}{n-1}, \quad\left(j=1,2, \ldots, n_{d}\right),
$$

where $n_{d}$ is the size of the dimension and $x_{j \max }$ and $x_{j \mathrm{~min}}$ are the upper and lower bounds of the $j$ th dimension. Then, $R=\left(R_{1}, R_{2}, \ldots, R_{n d}\right)$ is dominating radius of each sample point.

(4) Obtain new sample points. When $\mathrm{AE}_{i}>E[\mathrm{AE}]$, the prediction uncertainty near ith sample point is greater than the average prediction uncertainty of the existing sample points. It means the degree of nonlinearity near $i$ th sample point is relatively large. So a sample point is randomly added within the dominating radius of $i$ th sample point with equal probability. In order to avoid the added sample point being too close to the existing sample points, the sample point that meets the following condition is not added to the sample database:

$\left|\left(X_{*(j)}-X_{\text {closest }(j)}\right)\right|<\frac{R_{j}}{10}, \quad\left(j=1,2, \ldots, n_{d}\right)$,

where $X_{*}$ stands for the point to be added and $X_{\text {closest }}$ represents the sample point closest to point $X_{*}$. Formula (6) means that if the sample points $X_{*}$ and $X_{\text {closest }}$ are too close, they will influence the condition of the correlation matrix of the surrogate model, so the added sample point should be invalid.

(5) If the value of $R^{2}$ is greater than the preset value $\eta$, the final surrogate model is obtained; otherwise update the surrogate model. The new acquired sample points are added to the sample database. The corresponding response values of these new sample points are calculated. Then the surrogate model is updated according to the current database of sample points. Calculate the determination coefficient $R^{2}$. If the value of $R^{2}$ is greater than the preset value $\eta$, the adaptive sampling process ends; otherwise, return to step 2.

In order to illustrate the feasibility of LEE strategy, the one-dimensional test function in [32] is selected and its expression is

$$
f(x)=(6 x-2)^{2} \sin (12 x-4), \quad x \in[0,1] .
$$

Figures 2-4 are initial Kriging model, the absolute errors, and the updated Kriging model. Figure 2 shows that the overall prediction accuracy of the initial Kriging surrogate model is low, and the local errors near point 5 and point 6 are very large. It can be seen from Figure 3 that errors of sample points 5 and 6 of the initial Kriging model exceed $E[A E]$, so random sample points are added in the dominating radius of points 5 and 6 . It can be seen from Figure 4 that the added Kriging surrogate model has higher prediction accuracy. After adding the sample points, the prediction error in this area is significantly reduced, and the prediction accuracy is higher, which proves the effectiveness and feasibility of adaptive sampling based on LEE strategy.

In order to prove the versatility of LEE strategy for different surrogate models, the RBF surrogate model is also constructed based on the existing sample points and their response values. Figures 5-7 are initial RBF model, the absolute errors, and the updated RBF model. It can be seen from Figure 5 that the overall prediction accuracy of the initial RBF surrogate model is low, and the local errors near points 1 and 6 are the largest. It can be seen from Figure 6 that local errors of sample points 1 and 6 of the initial RBF model exceed $E[\mathrm{AE}]$, so random sample points are added in the dominating radius of sample points 1 and 6 . It can be seen from Figure 7 that the overall prediction accuracy of updated RBF surrogate model with two new sample points has been greatly improved, which further proves the feasibility and versatility of adaptive sampling based on LEE strategy.

The proposed LEE strategy is also compared with another adaptive sampling strategy called the Maximin Scaled Distance Approach (MSDA) [31] through the classic test functions. The specific information of the test functions is shown in Table 1.

The initial Kriging and RBF surrogate models are established, respectively, according to a certain number of initial sample points. The proposed LEE strategy and MSDA are applied to improve the accuracy of surrogate models. The convergence condition is $R^{2}>0.8$. Comparison results of Kriging and RBF surrogate models are listed in Table 2.

It can be seen from Table 2 that when the numbers of initial sample points of the two methods are the same, the numbers of total sample points used by LEE strategy are less than MSDA's. At the same time, except for $\mathrm{CN}$ function, the final values of $R^{2}$ of the LEE strategy are greater than those of the MSDA in most functions, which means that surrogate models constructed by LEE strategy can achieve higher prediction accuracy than those constructed by MSDA.

3.2. The Ensemble of Adaptive Surrogate Models. In this section we construct the ensemble of surrogate models with LEE strategy. The flowchart is shown in Figure 8.

The main steps are as follows:

(1) Build Kriging and RBF surrogate models. Existing researches [8-12] prove that, in most cases, interpolation type (Kriging and $\mathrm{RBF}$ ) surrogate models are more suitable for engineering problems. Therefore, this paper chooses Kriging and RBF models to form the ensemble of surrogate models. Construct Kriging and RBF models by using the initial sample points. Then, obtain the predicted error sum of square (PRESS) [33], MAE, and $R^{2}$ values of Kriging and RBF models by applying $C V$ verification method (LOO-leave one method). The absolute errors (AEs) of each sample point of Kriging and RBF models are calculated. Since Forrester [34] has already proved that the surrogate model has better predictive ability when the coefficient of determination $R^{2}$ is greater than 0.8 , we use $R^{2}>0.8$ as convergence conditions. 


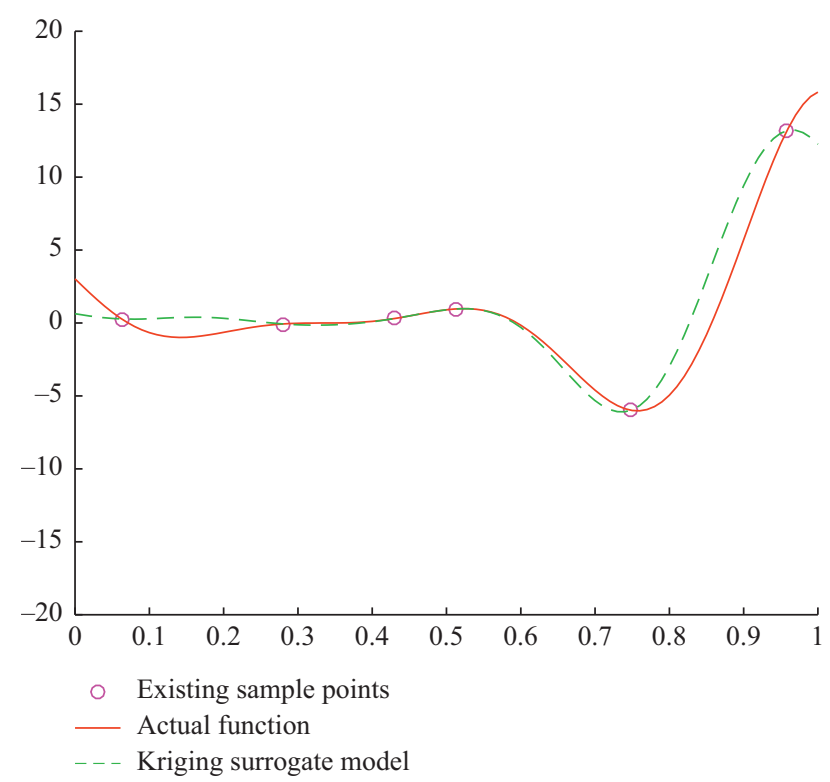

Figure 2: The initial Kriging model based on 6 sample points.

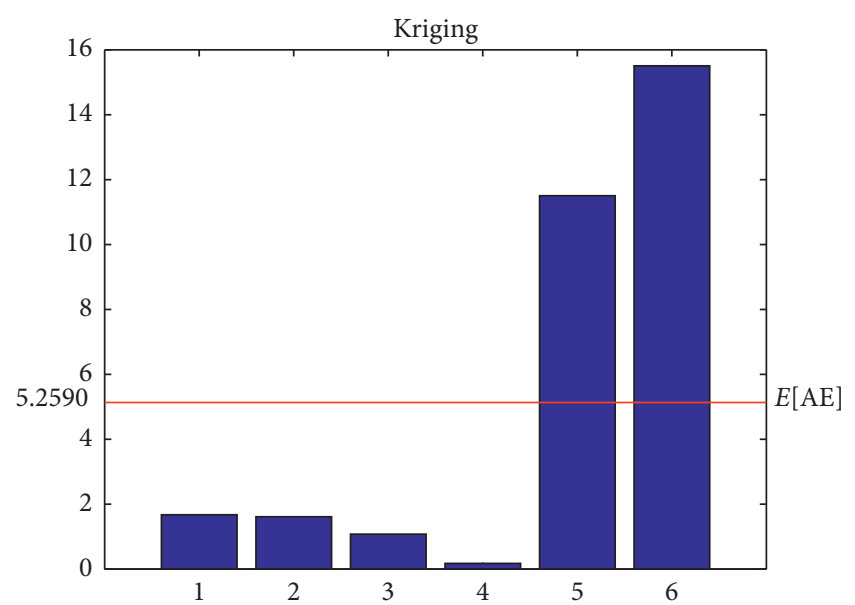

FIgURE 3: The absolute errors of samples of the initial Kriging model.

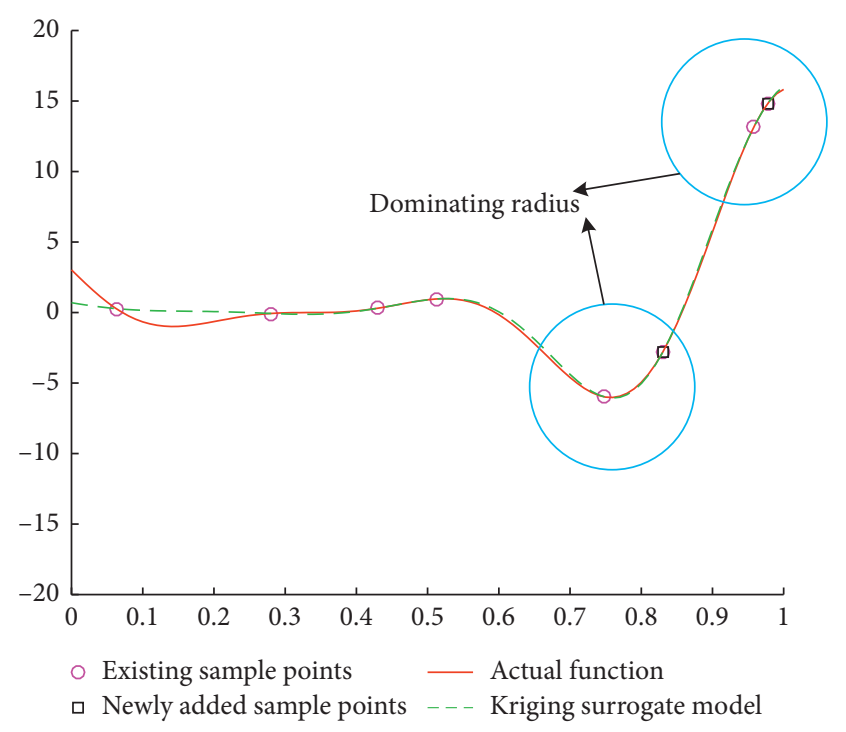

FIgUre 4: The updated Kriging model with 2 new sample points.

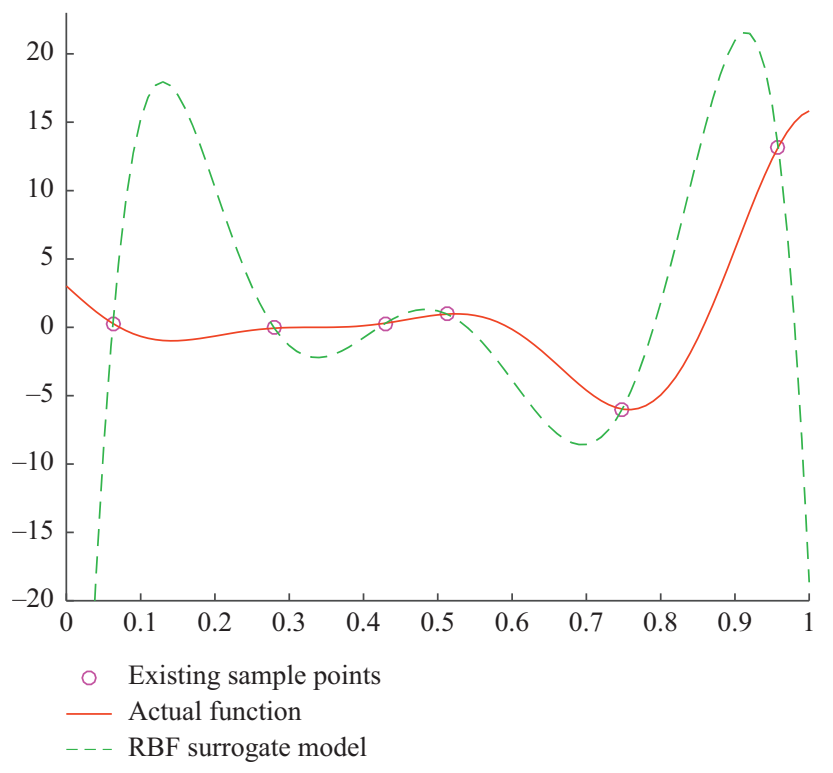

Figure 5: The initial RBF model based on 6 sample points.

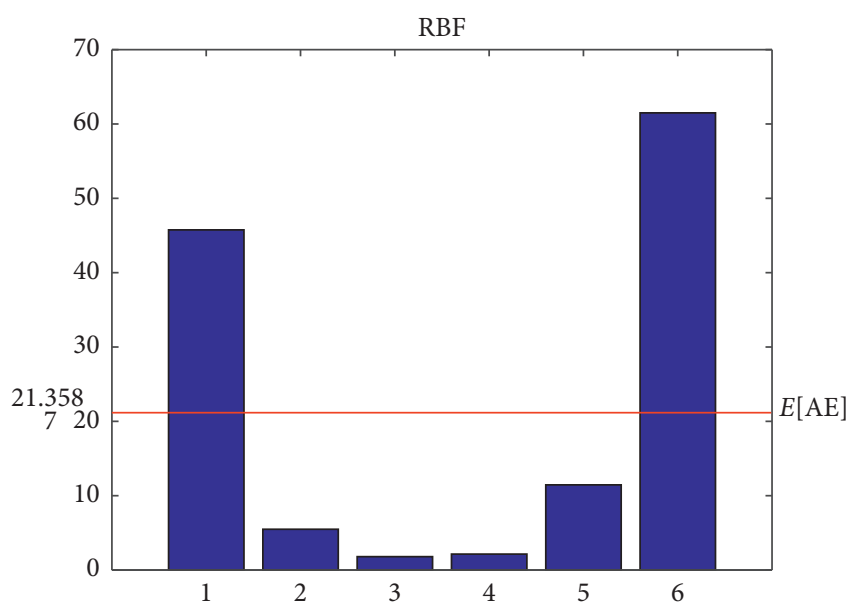

Figure 6: The absolute errors of samples of the initial RBF surrogate model.

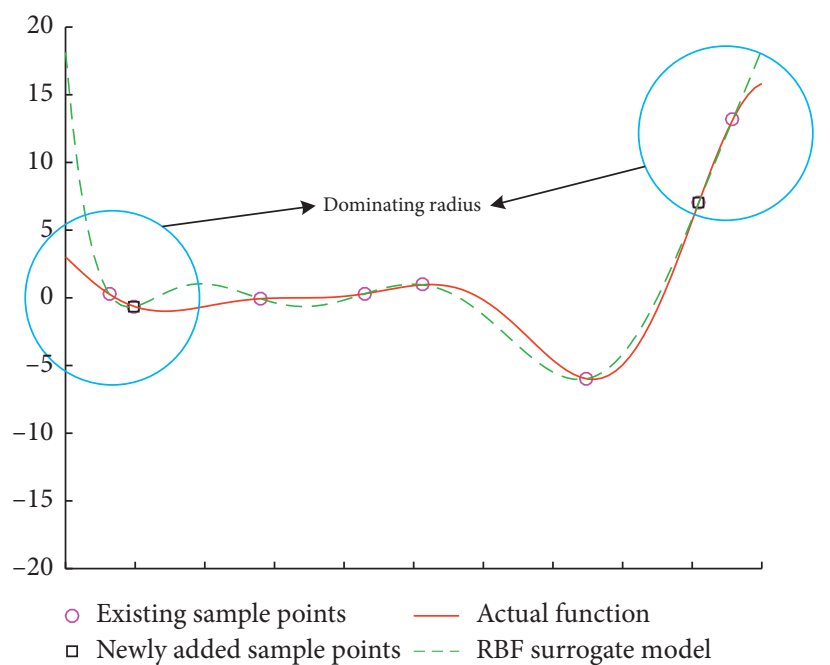

FIgURE 7: The updated RBF surrogate model with 2 new sample points. 
TABLE 1: Test function expression.

\begin{tabular}{|c|c|c|}
\hline Test function & Dimension & Test function expression \\
\hline Branin $(\mathrm{BN})$ & 2 & $\begin{array}{c}f(x)=\left(x_{2}-\left(5.1 / 4 \pi^{2}\right) x_{1}^{2}+(5 / \pi) x_{1}-6\right)^{2}+10(1-(1 / 8 \pi)) \cos \left(x_{1}\right)+10 \\
x_{1} \in[-5,10], x_{2} \in[0,15]\end{array}$ \\
\hline Hartmann3 (H3) & 3 & $\begin{array}{c}f(x)=-\sum_{i=1}^{4} \alpha_{i} \exp \left(-\sum_{j=1}^{3} A_{i j}\left(x_{j}-P_{i j}\right)^{2}\right) \\
\alpha=(1.0,1.2,3.0,3.2)^{T} \\
A=\left(\begin{array}{lll}3.0 & 10 & 30 \\
0.1 & 10 & 35 \\
3.0 & 10 & 30 \\
0.1 & 10 & 35\end{array}\right) \\
P=10^{-4}\left(\begin{array}{ccc}3689 & 1170 & 2673 \\
4699 & 4387 & 7470 \\
1091 & 8732 & 5547 \\
381 & 5743 & 8828\end{array}\right) \\
x_{i} \in[0,1]\end{array}$ \\
\hline Colville $(\mathrm{CV})$ & 4 & $\begin{array}{c}f(x)=100\left(x_{1}^{2}-x_{2}\right)^{2}+\left(x_{1}-1\right)^{2}+\left(x_{3}-1\right)^{2}+90\left(x_{3}^{2}-x_{4}\right)^{2} \\
+10.1\left(\left(x_{2}-1\right)^{2}+\left(x_{4}-1\right)^{2}\right)+19.8\left(x_{2}-1\right)\left(x_{4}-1\right) \\
x_{i} \in[-10,10], i=1,2\end{array}$ \\
\hline Six-Hump Camel (SHC) & 2 & $\begin{array}{c}f(x)=\left(4-2.1 x_{1}^{2}+\left(x_{1}^{4} / 3\right)\right) x_{1}^{2}+x_{1} x_{2}+\left(-4+4 x_{2}^{2}\right) x_{2}^{2} \\
x_{1} \in[-3,3], x_{2} \in[-2,2]\end{array}$ \\
\hline
\end{tabular}

TABLE 2: Comparison results of Kriging surrogate model.

\begin{tabular}{|c|c|c|c|c|c|c|}
\hline \multirow[b]{2}{*}{$\begin{array}{l}\text { Test } \\
\text { function }\end{array}$} & \multirow[b]{2}{*}{ Approach } & \multirow[b]{2}{*}{$\begin{array}{c}\text { The number of initial } \\
\text { samples }\end{array}$} & \multicolumn{2}{|c|}{ Kriging model } & \multicolumn{2}{|c|}{ RBF model } \\
\hline & & & $\begin{array}{c}\text { The number of total } \\
\text { samples }\end{array}$ & $\begin{array}{l}\text { Final value of } \\
R^{2}\end{array}$ & $\begin{array}{c}\text { The number of total } \\
\text { samples }\end{array}$ & $\begin{array}{l}\text { Final value of } \\
R^{2}\end{array}$ \\
\hline \multirow{2}{*}{$\mathrm{BN}$} & LEE & \multirow{2}{*}{10} & 18 & 0.946 & 15 & 0.908 \\
\hline & MSDA & & 27 & 0.899 & 27 & 0.873 \\
\hline \multirow{2}{*}{$\mathrm{H} 3$} & LEE & \multirow{2}{*}{15} & 26 & 0.896 & 35 & 0.902 \\
\hline & MSDA & & 34 & 0.837 & 39 & 0.879 \\
\hline \multirow{2}{*}{$\mathrm{CV}$} & LEE & \multirow{2}{*}{20} & 29 & 0.909 & 30 & 0.934 \\
\hline & MSDA & & 44 & 0.943 & 41 & 0.901 \\
\hline \multirow{2}{*}{ SHC } & LEE & \multirow{2}{*}{10} & 25 & 0.920 & 21 & 0.941 \\
\hline & MSDA & & 36 & 0.883 & 29 & 0.866 \\
\hline
\end{tabular}

(2) Obtain new sample points. The new sample points are generated by applying adaptive sampling method based on LEE strategy. The sample database is updated.

(3) Update the Kriging and the RBF models. Calculate the true response values of the newly added sample points and reconstruct the Kriging and the RBF models. As long as the $R^{2}$ of one of the two surrogate models is greater than 0.8 , the operation of adding sample points is ended, and the final Kriging model and RBF model are obtained. Otherwise return to step 2.

(4) Calculate the weight coefficients of the Kriging and the RBF models and get the final ensemble of adaptive surrogate models. Cross validation (CV) [35] is performed to obtain the respective PRESS values of Kriging and RBF models. When there are $n$ sample points in the database, all sample points except the $i$ th point are used to construct the single surrogate model, and the $i$ th point is used as a test point. The prediction error of the $i$ th sample point is

$$
e_{i}=y_{i}-\hat{y}_{-i}
$$

where $y_{i}$ is the true response value of the $i$ th sample point and $\widehat{y}_{-i}$ is the predicted response value of the $i$ th sample point in the single surrogate model composed of all sample points except $i$ th sample point. The prediction sum of squares is the sum of the prediction errors of all sample points, as shown in the following formula:

$$
\text { PRESS }=\sum_{i=1}^{n} e_{i}^{2}
$$

The weight coefficient corresponding to each single surrogate model is calculated by the inverse proportional 


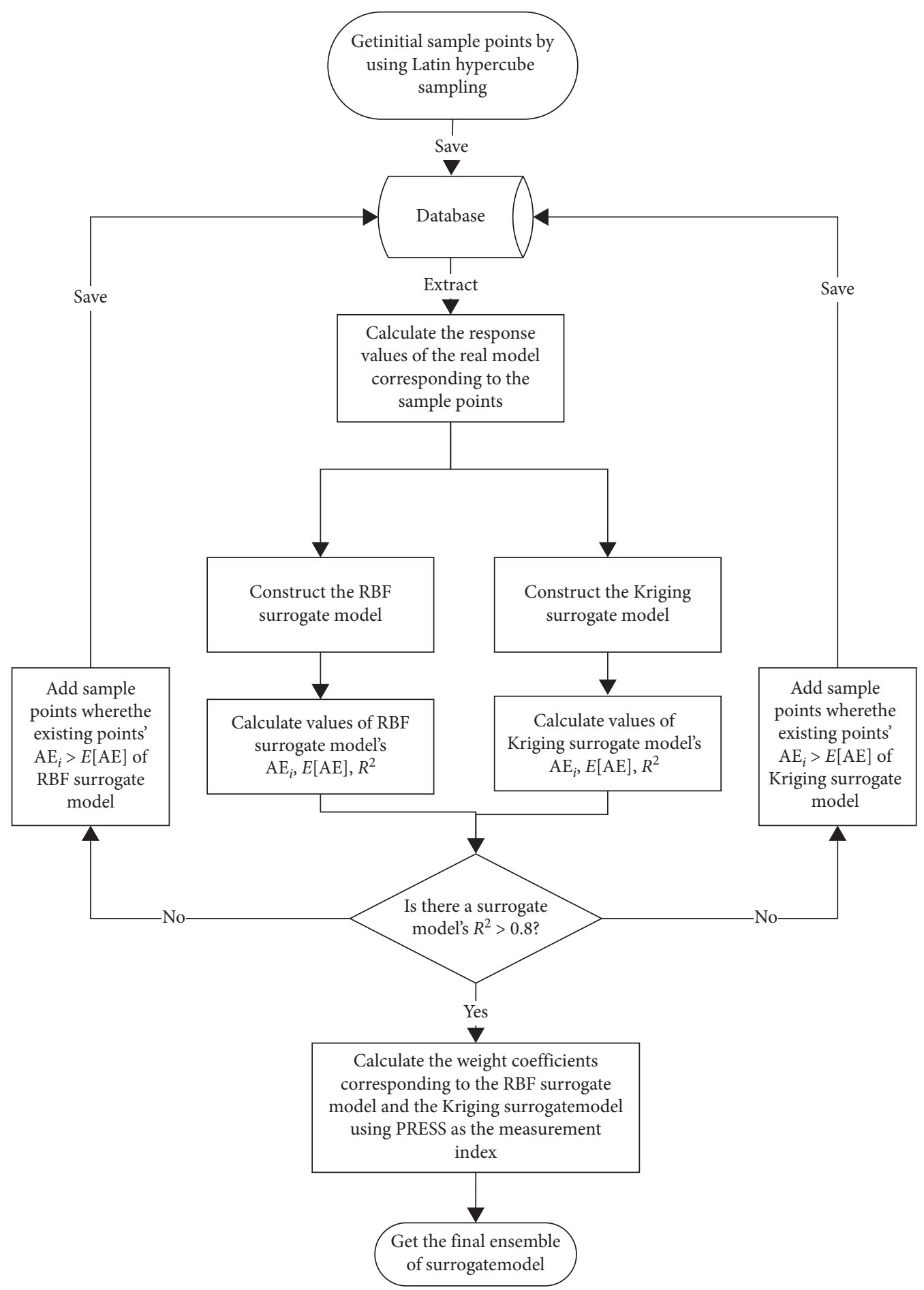

FIgURE 8: The construction of the ensemble of adaptive surrogate model based on LEE strategy.

averaging method, and the weight coefficient calculation formula is

$$
\omega_{i}=\frac{\left(1 / P_{i}\right)}{\sum_{j=1}^{N}\left(1 / P_{j}\right)}
$$

where $P_{i}$ is the PRESS value at the $i$ th sample point. In this paper, $N$ is equal to 2 . Then the final ensemble of adaptive surrogate models is obtained by linearly weighting each surrogate model.

\section{Numerical Example Analysis}

In order to verify the versatility and effectiveness of the ensemble of adaptive surrogate models based on local error 
expectations, we compare the proposed method (ensemble of adaptive surrogate model, EOASM) with three typical ensemble of surrogate model construction methods: PRESS method, BestPRESS method, and PWS (PRESS Weighted Surrogate) method [36].

Among the three most widely used methods for constructing an ensemble of surrogate model, the most classic one is to use PRESS as a measure of the weight coefficient calculation. If the PRESS value of a certain surrogate model is larger, the weight coefficient is smaller, also known as an inverse proportional averaging method, and its weight coefficient calculation formula is

$$
\omega_{i}=\frac{\left(1 / P_{i}\right)}{\sum_{j=1}^{N}\left(1 / P_{j}\right)} .
$$

The BestPRESS method selects the single surrogate model with the smallest PRESS value as the final surrogate model, which is essentially a single surrogate model. Another method is the heuristic calculation weight coefficient algorithm proposed by Goel [36], and its calculation formula is

$$
\omega_{i}=\frac{\omega_{i}^{*}}{\sum_{j=1}^{n} \omega_{j}^{*}},
$$

where $\omega_{i}^{*}=\left(E_{i}+\alpha E_{\text {avg }}\right)^{\beta}$ and $E_{\text {avg }}=\left(\sum_{j=1}^{n} E_{j}\right) / n$. $E_{i}$ is the PRESS of the $i$ th surrogate model. The recommended parameter values are $\alpha=0.05, \beta=-1$.

4.1. Benchmark Functions. In this paper, six benchmark functions from low dimension to high dimension are selected. The information of benchmark functions is shown in Table 3.

The Branin, Hartmann-3, and Hartmann-4 functions are low-dimensional. Latin hypercube sampling with $5 n$ sample points is enough, which meet the accuracy requirements. Since the Hartmann-6, Styblinski-Tang8, and StyblinskiTang10 are high dimensional, the Latin hypercube sampling with $20 n$ sample points is used.

4.2. The Analysis of Global Prediction Accuracy. The global prediction accuracies of different ensembles of surrogate models are compared. The total number of samples is recorded when the EOASM method reaches the convergence condition. For the other three ensembles of surrogate models constructed by the PRESS method, BestPRESS method, and PWS method, the Latin hypercube sampling method is used to generate the same total sample size. So the number of sample points in the four methods is the same. After 20 comparative experiments, the average values of the determination of coefficient $R^{2}$ of each ensemble of surrogate models are shown in Table 4 .

It can be seen from Table 4 that when the total number of sample points is the same, the prediction accuracy of the ensemble of surrogate model constructed by the EOASM method is the highest. For example, for the Branin function, the average value of determination coefficient $R^{2}$ of EOASM is 0.9446. Among the other three ensembles of surrogate models, the PRESS method has the largest average value of $R^{2}$, which is much lower than that of the EOASM method. The results of the other test functions are similar to the Branin function.

4.3. The Analysis of Local Prediction Accuracy. The maximum absolute error (MAE) is used to evaluate the local accuracy. The maximum absolute error of the ensemble of surrogate model constructed by each method is compared when the number of sample points is the same. Table 5 shows the mean values of MAE of different ensembles of surrogate models.

It can be seen from 6 benchmark functions that EOASM method has the smallest average value of the MAE among four ensembles of surrogate models, which means that the proposed method has the highest predict accuracy among four methods.

4.4. Robustness Analysis. Robustness is an important indicator for evaluating surrogate models. The robustness refers to the insensitivity of the prediction accuracy of the surrogate model to random sampling of sample points. In order to compare the robustness of each surrogate model intuitively, 20 sampling experiments are performed for each benchmark function. The distribution results of the determination coefficient $R^{2}$ are presented in box plot [37], which are shown in Figure 9.

In Figure 9, the box length indicates whether the surrogate model's determination coefficient $R^{2}$ fluctuates greatly. The smaller the box length, the stronger the robustness of the surrogate model. It can be clearly seen that the box length of the ensemble of surrogate model constructed by the EOASM method is the shortest in each benchmark function, which indicates the EOASM method has the strongest robustness.

\section{Engineering Application}

In the design of the palletizing robot, the design of the driving arm base plays a key role. The overall assembly of the palletizing robot is shown in Figure 10.

The driving arm base bears large load. When it is assembled with the boom, it will deform to a certain extent, which will cause strain and stress. However, these physical quantities are difficult to express using explicit functions. It is often necessary to obtain their data through a large number of simulation tests. The specific material properties are shown in Table 6.

The structure of the driving arm base is shown in Figure 11. Considering the assembly relationship of each part, four nonassembly dimensions are selected as design variables, which are shown in Table 7 . When the force and torque of the driving arm base reach the maximum, the generated stress is the largest. The fatigue damage is more likely to be caused. Power is carried out through UG software simulation to obtain the maximum force and torque of the assembly hole of the driving arm base. 
TABle 3: Test function expression.

\begin{tabular}{|c|c|c|}
\hline Test function expression & Dimension & Test function expression \\
\hline Branin & 2 & $\begin{array}{c}f(x)=\left(x_{2}-\left(5.1 / 4 \pi^{2}\right) x_{1}^{2}+(5 / \pi) x_{1}-6\right)^{2}+10(1-(1 / 8 \pi)) \cos \left(x_{1}\right)+10 \\
x_{1} \in[-5,10], x_{2} \in[0,15]\end{array}$ \\
\hline Hartmann-3 & 3 & $\begin{array}{c}f(x)=-\sum_{i=1}^{4} \alpha_{i} \exp \left(-\sum_{j=1}^{3} A_{i j}\left(x_{j}-P_{i j}\right)^{2}\right) \\
\alpha=(1.0,1.2,3.0,3.2)^{T} \\
A=\left(\begin{array}{lll}3.0 & 10 & 30 \\
0.1 & 10 & 35 \\
3.0 & 10 & 30 \\
0.1 & 10 & 35\end{array}\right) \\
P=10^{-4}\left(\begin{array}{ccc}3689 & 1170 & 2673 \\
4699 & 4387 & 7470 \\
1091 & 8732 & 5547 \\
381 & 5743 & 8828\end{array}\right) \\
x_{i} \in[0,1]\end{array}$ \\
\hline Hartmann-4 & 4 & $\begin{array}{c}f(x)=(1 / 0.839)\left[1.1-\sum_{i=1}^{4} \alpha_{i} \exp \left(-\sum_{j=1}^{4} A_{i j}\left(x_{j}-P_{i j}\right)^{2}\right)\right] \\
\mid \alpha=(1.0,1.2,3.0,3.2)^{T} \\
A=\left(\begin{array}{cccccc}10 & 3 & 17 & 3.5 & 1.7 & 8 \\
0.05 & 10 & 17 & 0.1 & 8 & 14 \\
3 & 3.5 & 1.7 & 10 & 17 & 8 \\
17 & 8 & 0.05 & 10 & 0.1 & 14\end{array}\right) \\
P=10^{-4}\left(\begin{array}{cccccc}1312 & 1696 & 5569 & 124 & 8283 & 5886 \\
2329 & 4135 & 8307 & 3736 & 1004 & 9991 \\
2348 & 1451 & 3522 & 2883 & 3047 & 6650 \\
4047 & 8828 & 8732 & 5743 & 1091 & 381\end{array}\right) \\
x \in[0,1]\end{array}$ \\
\hline \multirow[b]{2}{*}{ Hartmann-6 } & \multirow[b]{2}{*}{6} & $\begin{array}{c}f(x)=-\sum_{i=1}^{4} \alpha_{i} \exp \left(-\sum_{j=1}^{6} A_{i j}\left(x_{j}-P_{i j}\right)^{2}\right) \\
\alpha=(1.0,1.2,3.0,3.2)^{T}\end{array}$ \\
\hline & & $\begin{aligned} A & =\left(\begin{array}{cccccc}10 & 3 & 17 & 3.5 & 1.7 & 8 \\
0.05 & 10 & 17 & 0.1 & 8 & 14 \\
3 & 3.5 & 1.7 & 10 & 17 & 8 \\
17 & 8 & 0.05 & 10 & 0.1 & 14\end{array}\right) \\
P=10^{-4} & \left(\begin{array}{ccccccc}1312 & 1696 & 5569 & 124 & 8283 & 5886 \\
2329 & 4135 & 8307 & 3736 & 1004 & 9991 \\
2348 & 1451 & 3522 & 2883 & 3047 & 6650 \\
4047 & 8828 & 8732 & 5743 & 1091 & 381\end{array}\right) \\
x_{i} \in[0,1] & \end{aligned}$ \\
\hline $\begin{array}{l}\text { Styblinski-Tang } 8 \\
\text { Styblinski-Tang10 }\end{array}$ & $\begin{array}{c}8 \\
10\end{array}$ & $\begin{array}{l}f(x)=(1 / 2) \sum_{i=1}^{8}\left(x_{i}^{4}-16 x_{i}^{2}+5 x_{i}\right), x_{i} \in[-5,5] \\
f(x)=(1 / 2) \sum_{i=1}^{10}\left(x_{i}^{4}-16 x_{i}^{2}+5 x_{i}\right), x_{i} \in[-5,5]\end{array}$ \\
\hline
\end{tabular}

TABLE 4: Mean values of $R^{2}$.

\begin{tabular}{|c|c|c|c|c|c|}
\hline Benchmark test function & Total sample & PRESS & BestPRESS & PWS & EOASM \\
\hline Branin & 15 & 0.7351 & 0.7089 & 0.7364 & 0.9446 \\
\hline Hartmann-3 & 23 & 0.6934 & 0.6643 & 0.6935 & 0.9007 \\
\hline Hartmann-4 & 30 & 0.6549 & 0.5847 & 0.6547 & 0.9313 \\
\hline Hartmann-6 & 189 & 0.6884 & 0.6612 & 0.6883 & 0.9797 \\
\hline Styblinski-Tang8 & 240 & 0.4310 & 0.3903 & 0.4413 & 0.9514 \\
\hline Styblinski-Tang10 & 299 & 0.2931 & 0.2588 & 0.2931 & 0.9624 \\
\hline
\end{tabular}

The curve of the force and torque with time is shown in Figure 12. It can be seen that, at 3 seconds, the driving arm base bears the maximum force and the maximum torque.
Since the maximum stress is difficult to calculate directly, it is selected as the object function, and its true response value is obtained by simulation with Ansys finite element software, as shown in Figure 13. 
TABle 5: Mean values of MAE.

\begin{tabular}{|c|c|c|c|c|c|}
\hline Benchmark test function & Total sample & PRESS & BestPRESS & PWS & EOASM \\
\hline Branin & 15 & 108.8272 & 83.2561 & 108.7975 & 38.8241 \\
\hline Hartmann-3 & 23 & 1.2506 & 1.1722 & 1.2516 & 0.9772 \\
\hline Hartmann-4 & 30 & 2.2512 & 2.0696 & 2.2534 & 0.7489 \\
\hline Hartmann-6 & 189 & 0.4521 & 0.6021 & 0.4524 & 0.1578 \\
\hline Styblinski-Tang8 & 240 & 370.6343 & 331.6834 & 370.5411 & 91.3723 \\
\hline Styblinski-Tang10 & 299 & 330.5281 & 308.0860 & 330.7615 & 76.8565 \\
\hline
\end{tabular}

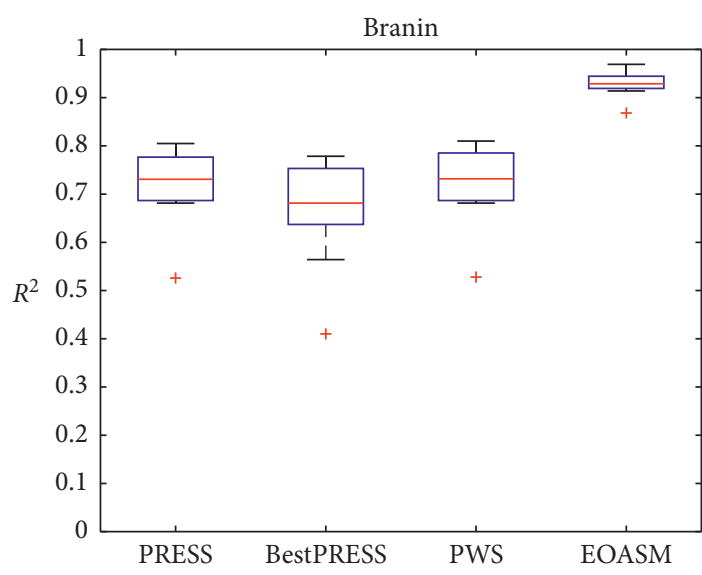

(a)

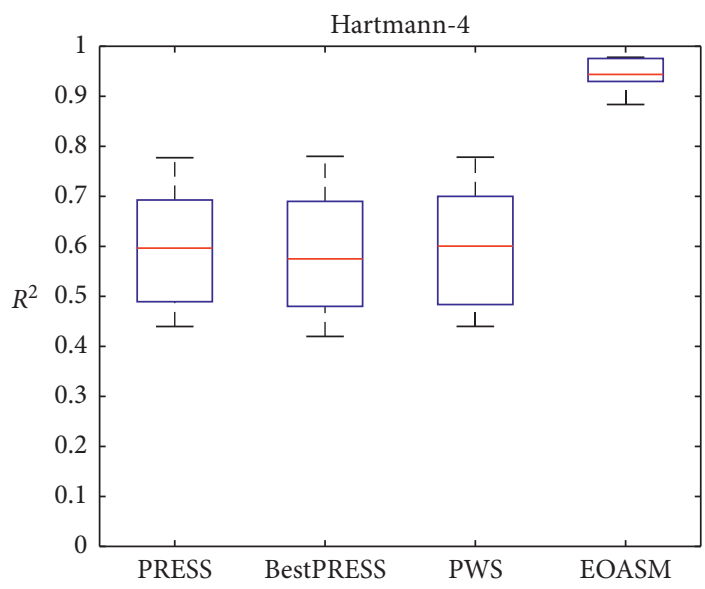

(c)

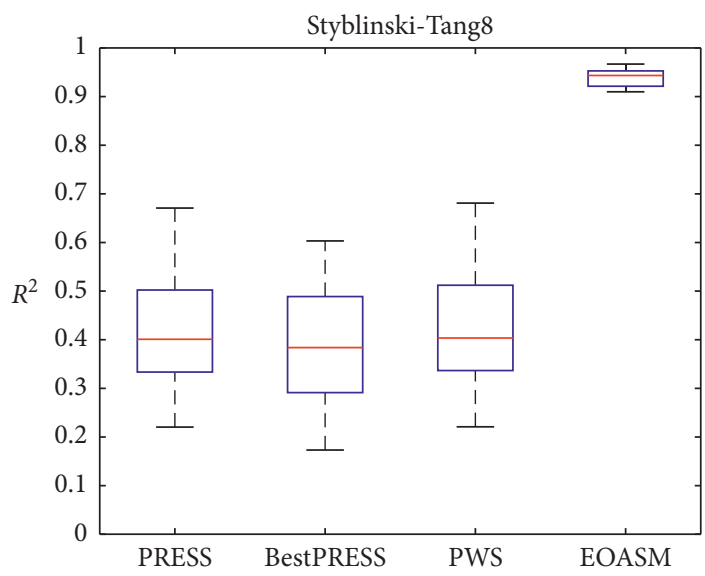

(e)

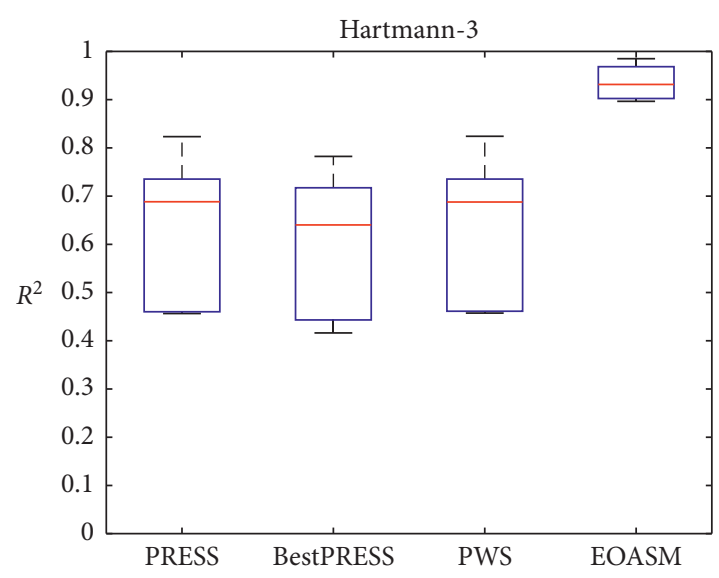

(b)

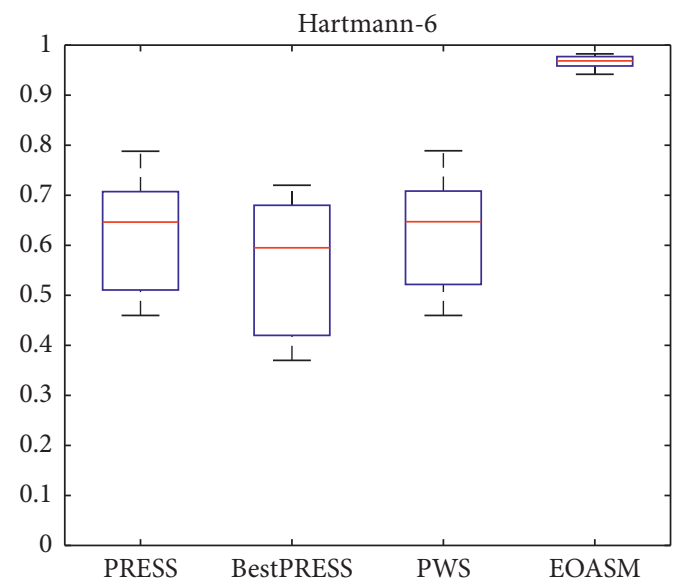

(d)

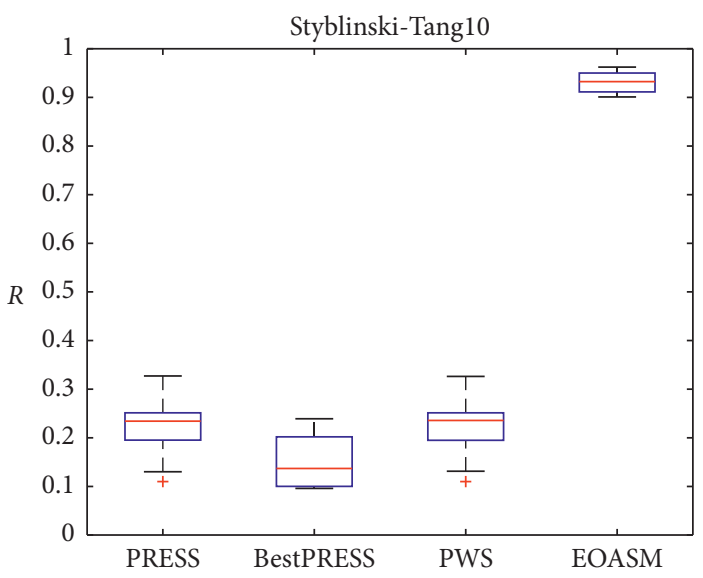

(f)

Figure 9: $R^{2}$ box diagram of the ensembles of surrogate models. (a) Branin function, (b) Hartmann-3 function, (c) Hartmann-4 function, (d) Hartmann-6 function, (e) Styblinski-Tang8 function, and (f) Styblinski-Tang10 function. 


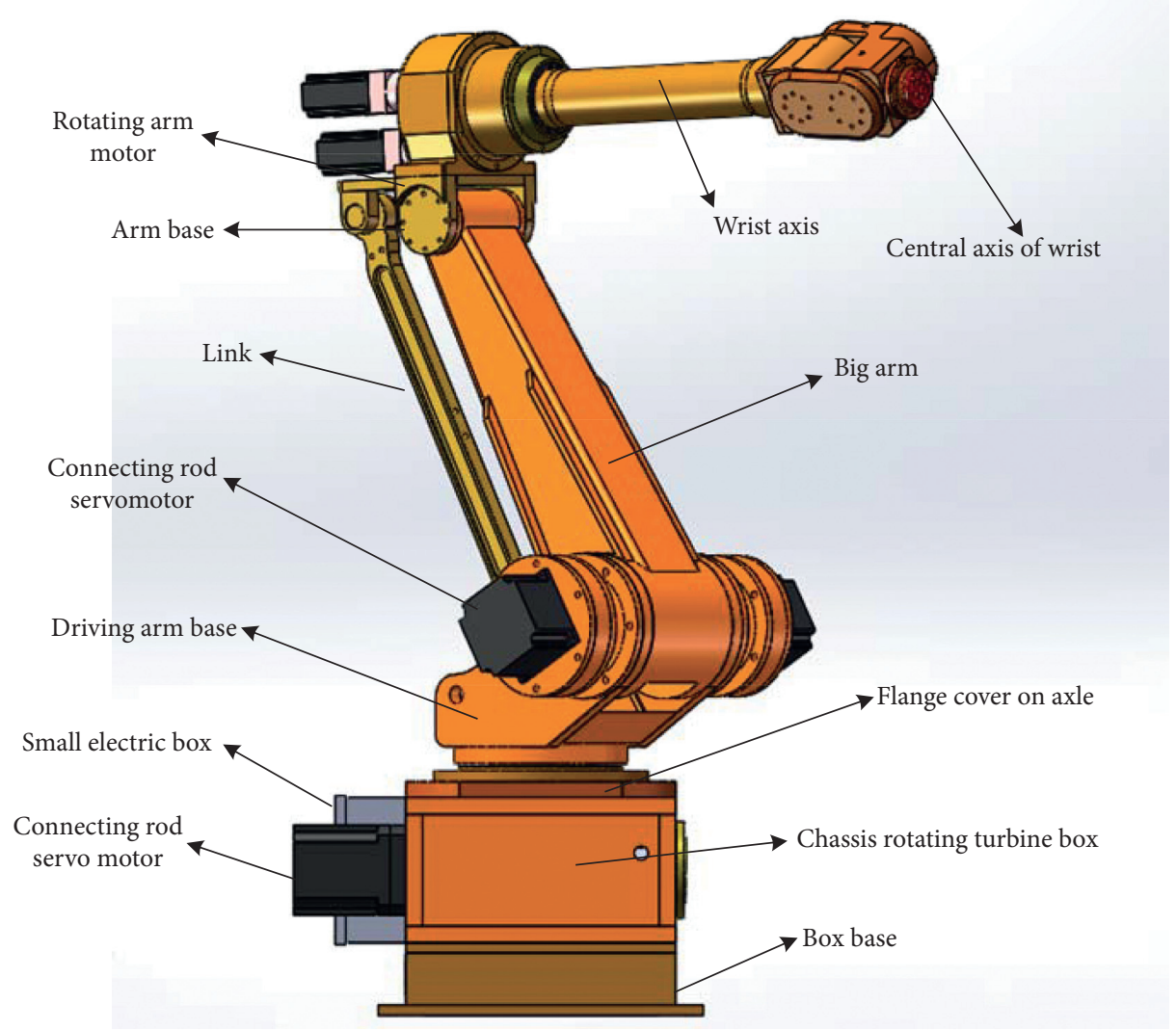

FIGURE 10: Overall assembly drawing of palletizing robot.

TABLE 6: Material properties of QT500-7.

\begin{tabular}{lcc}
\hline Physical quantity & Unit & Value \\
\hline Density & $\mathrm{kg} / \mathrm{m}^{3}$ & $7 \times 10^{3}$ \\
Elastic modulus & $\mathrm{Pa}$ & $1.62 \times 10^{11}$ \\
Poisson's ratio & - & 0.28 \\
Yield strength & $\mathrm{Pa}$ & $3.2 \times 10^{8}$ \\
Tensile strength & $\mathrm{Pa}$ & $5 \times 10^{8}$ \\
Shear modulus & $\mathrm{Pa}$ & $6.27 \times 10^{10}$ \\
\hline
\end{tabular}

The proposed method in this paper is used to construct the ensemble of surrogate model of maximum stress. The Latin hypercube sampling is initially adopted. The number of initial sample points is $10 n_{d}$, which is 40 sample points.

The values of global accuracy evaluation index $R^{2}$ and the local accuracy evaluation index MAE of surrogate model constructed by the EOASM method are shown in Table 8. It can be seen that the number of total sample points after convergence is 60 . The CPU of the simulation platform is Intel Core $15-45903.30 \mathrm{GHz}$, the memory is $16 \mathrm{G}$, and the operating system is Windows 10 . It takes 6 minutes to perform a static structural simulation. The traditional design requires thousands of simulation experiments to roughly find the optimal value; optimization based on surrogate model only requires 60 simulation experiments, which greatly reduces computational cost of the simulation. The

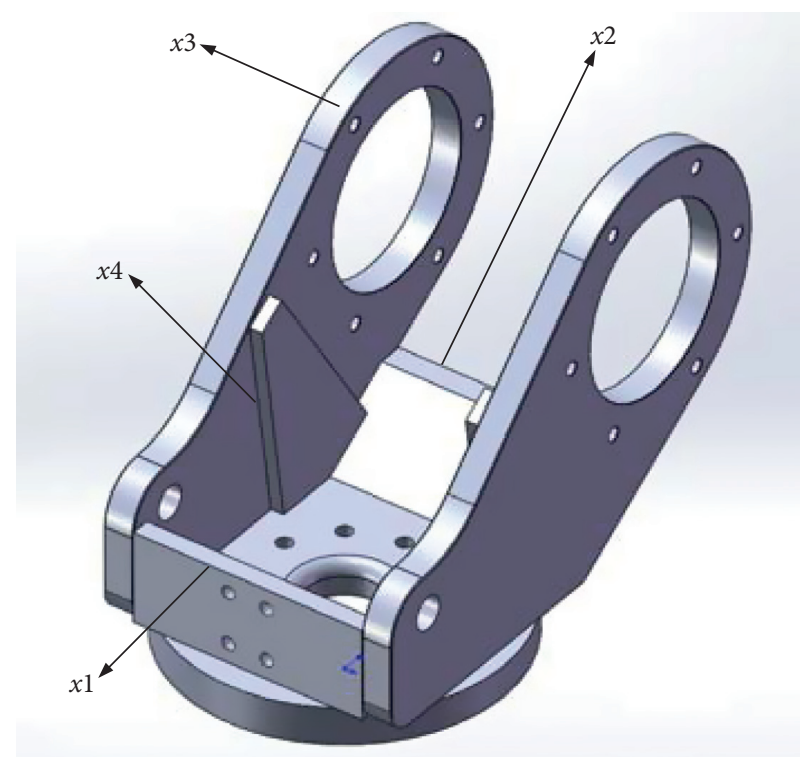

FIGURE 11: Driving arm base of palletizing robot.

initial value of $R^{2}$ increases from 0.3822 to 0.8979 . The global prediction accuracy is increased by $135 \%$. Meanwhile, the value of MAE reduces from 4.1565 to 0.5007 . The local 
TABLe 7: Design variables of driving arm base.

\begin{tabular}{lccc}
\hline Design variables & Name & Unit & Ranges \\
\hline$x_{1}$ & Thickness of front plate & $\mathrm{mm}$ & $13-18$ \\
$x_{2}$ & Thickness of back plate & $\mathrm{mm}$ & $8-13$ \\
$x_{3}$ & Thickness of left and right board & $\mathrm{mm}$ & $20-25$ \\
$x_{4}$ & Thickness of rib & $\mathrm{mm}$ & $8-13$ \\
\hline
\end{tabular}

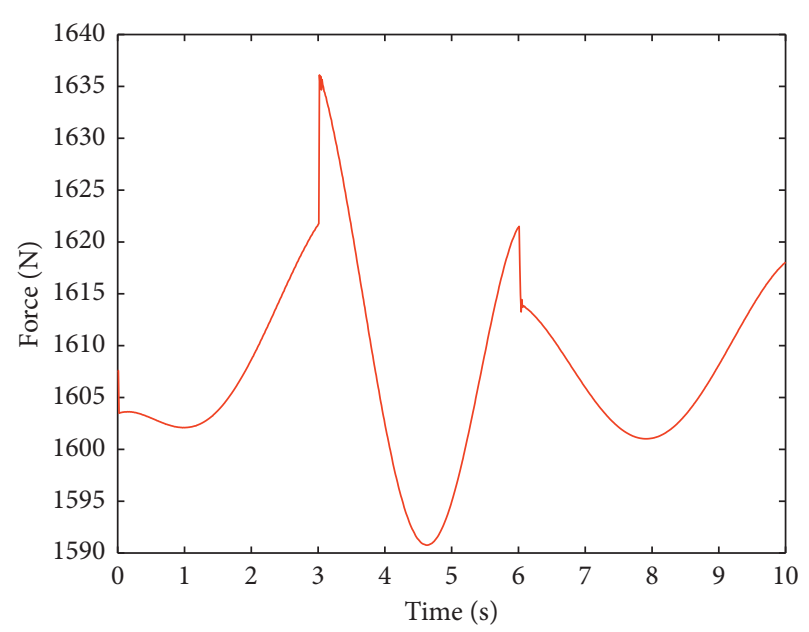

(a)

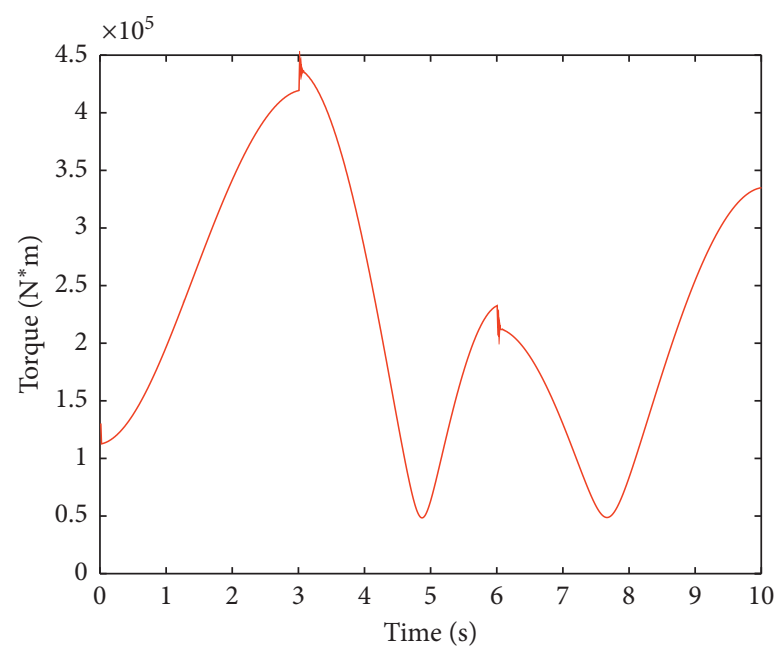

(b)

Figure 12: The force and torque of the driving arm base. (a) The force changes with time. (b) The torque changes with time.

\begin{tabular}{|l}
$6.8438 \mathrm{Max}$ \\
6.0836 \\
5.3234 \\
4.5632 \\
3.003 \\
3.0420 \\
2.2826 \\
1.5224 \\
0.76224 \\
$0.0020469 \mathrm{Min}$
\end{tabular}

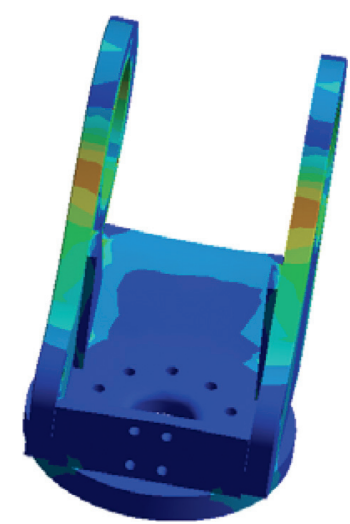

FIGURE 13: Stress cloud diagram of driving arm base.

TABle 8: Prediction accuracy of ensemble of surrogate model constructed by EOASM method.

\begin{tabular}{lcccc}
\hline $\begin{array}{l}\text { Evaluation } \\
\text { perspective }\end{array}$ & $\begin{array}{c}\text { The initial } \\
\text { samples }\end{array}$ & $\begin{array}{c}\text { The total } \\
\text { samples }\end{array}$ & $\begin{array}{c}\text { Initial } \\
\text { data }\end{array}$ & $\begin{array}{c}\text { EOASM } \\
\text { data }\end{array}$ \\
\hline $\begin{array}{l}R^{2} \text { average } \\
\text { MAE average }\end{array}$ & 40 & 60 & 0.3822 & 0.8979 \\
& & & 4.1565 & 0.5007 \\
\hline
\end{tabular}

prediction accuracy is significantly improved. In summary, the EOASM method has good applicability to engineering problems and can greatly reduce the calculation cost of physical experiments.

\section{Conclusion}

(1) The adaptive sampling based on LEE strategy can greatly improve the prediction accuracy of the surrogate model based on as few sample points as possible, and it also has strong applicability to different types of surrogate models.

(2) The EOASM method based on LEE strategy can greatly improve the global prediction accuracy, local prediction accuracy, and the robustness of the ensemble of surrogate models. 
(3) Although the prediction accuracy and robustness of the ensemble of surrogate models constructed by the EOASM method have been improved to some extent, it still has not escaped the high-dimensional curse of the surrogate model. Under the condition that the sample size is already large, it is possible that the accuracy of the surrogate model is extremely low. Therefore, the high-dimensional problem of the surrogate model is still a problem to be solved.

\section{Data Availability}

The data used to support the findings of this paper are included within the article (Table 2).

\section{Conflicts of Interest}

The authors declare that they have no conflicts of interest.

\section{Acknowledgments}

This research was supported by the National Natural Science Foundation of China under the Contract no. 51975106.

\section{References}

[1] P. Palar and K. Shimoyama, "On efficient global optimization via universal Kriging surrogate models," Structural \& Multidisciplinary Optimization, vol. 14, pp. 329-335, 2017.

[2] S. W. Liu, J. M. Sun, X. Gao et al., "Analysis and establishment of drilling rate prediction model based on artificial neural network," Computer Science, vol. 14, no. 6, pp. 605-608, 2019.

[3] S. Ozcanan and A. O. Atahan, "RBF surrogate model and EN1317 collision safety-based optimization of two guardrails," Structural and Multidisciplinary Optimization, vol. 60, no. 1, pp. 343-362, 2019.

[4] W. Wang, F. Dou, X. Yu et al., "Load forecasting method based on SVR under electricity market reform," IOP Conference Series Earth and Environmental, vol. 467, Article ID 012201, 2020.

[5] J. S. Cheng and H. Yu, "Optimal design of ossicle muffler based on response surface method," Journal of Hunan University: Natural Science Edition, vol. 44, no. 2, pp. 60-65, 2017.

[6] H. J. Huang, B. W. Zhang, G. Q. Wu et al., "Multidisciplinary design optimization of car body based on the ensemble of surrogate model," Auto Motive Engineering, vol. 38, no. 9, pp. 1107-1113, 2016.

[7] L. Yan, X. J. Duan, B. W. Liu et al., "Weighted surrogate model based on Kullback-Leibler distance dispersion," Journal of National University of Defense Technology, vol. 41, no. 3, pp. 159-165, 2019.

[8] Z. M. Lu, L. Q. Wang, J. Zhao et al., “An optimization method of mixed integer programming based on multi-surrogate model," Control and Decision, vol. 34, no. 2, pp. 362-368, 2019.

[9] F. Pan, Research on the Ensemble of Surrogate Model Method and its Application in Lightweight Design of Car Body, Shanghai Jiaotong University, Shanghai, China, 2011.

[10] X. Liu, Research on Structural Optimization Design of Body Parts Based on Robust Design, Hunan University, Hunan, China, 2013.

[11] Z. Xing, R. Qu, Y. Zhao, Q. Fu, Y. Ji, and W. Lu, "Identifying the release history of a groundwater contaminant source based on an ensemble surrogate model," Journal of Hydrology, vol. 572, pp. 501-516, 2019.

[12] J. Yin and T. C. F.. Tsai, "Bayesian set pair analysis and machine learning based ensemble surrogates for optimal multi-aquifer system remediation design," Journal of $\mathrm{Hy}$ drology, vol. 580, pp. 411-426, 2020.

[13] F. Li, X. Cai, and L. Gao, "Ensemble of surrogates assisted particle swarm optimization of medium scale expensive problems," Applied Soft Computing, vol. 580, pp. 74-79, 2018.

[14] F. O. Donncha, Y. Zhang, B. Chen et al., "Ensemble model aggregation using a computationally lightweight machinelearning model to forecast ocean waves," Journal of Marine Systems, vol. 199, pp. 361-378, 2019.

[15] L. Ouyang, L. Wan, C. Park, J. Wang, and Y. Ma, "Ensemble RBF modeling technique for quality design," Journal of Management Science and Engineering, vol. 4, no. 2, pp. 105118, 2019.

[16] L. Chen, H. Qiu, C. Jiang, X. Cai, and L. Gao, "Ensemble of surrogates with hybrid method using global and local measures for engineering design," Structural and Multidisciplinary Optimization, vol. 57, no. 4, pp. 1711-1729, 2018.

[17] J. Zhang, X. Yue, J. Qiu et al., "A unified ensemble of surrogates with global and local measures for global metamodeling," Engineering Optimization, vol. 57, no. 1, pp. 1-22, 2020.

[18] L. Xia and D. Wang, "Aerodynamic optimization method based on Kriging adaptive surrogate model," Aeronautical Computing Technology, vol. 57, no. 1, pp. 17-21, 2013.

[19] N. C. Xiao, K. Yuan, and Y. S. Wang, "Structural reliability analysis method based on sequence surrogate model," Journal of University of Electronic Science and Technology of China, vol. 48, no. 1, pp. 156-160, 2019.

[20] Z. H. Han, Y. Zhang, C. Z. Xu et al., "Aerodynamic optimization design of large civil aircraft wings based on surrogate model," Journal of Aeronautics, vol. 40, no. 1, pp. 150-165, 2019.

[21] R. Mukesh, P. Soma, V. Karthikeyan et al., "Prediction of ionospheric vertical total electron content from GPS data using ordinary kriging-based surrogate model," Astrophysics \& Space Science, vol. 364, no. 1, pp. 15-18, 2019.

[22] G. J. Zhang, Adaptive Optimization Algorithm Based on MultiSurrogate Model and its Application in Wheel Bearing Shaft Riveting, South China University of Technology, Beijing, China, 2016.

[23] B. Y. Peng, Application of Optimization Algorithm Based on Hybrid Surrogate Model in Electromagnetic Equipment Design, Shenyang University of Technology, Beijing, China, 2019.

[24] S. M. Sanchez and P. J. Sanchez, "Very large fractional factorial and central composite designs," ACM Transactions on Modeling and Computer Simulation, vol. 15, no. 4, pp. 362377, 2005.

[25] J. Wang, J. Liu, G. Zhang, J. Zhou, and K. Cen, "Orthogonal design process optimization and single factor analysis for bimodal acoustic agglomeration," Powder Technology, vol. 210, no. 3, pp. 315-322, 2011.

[26] D. Clifford, J. E. Payne, M. J. Pringle, R. Searle, and N. Butler, "Pragmatic soil survey design using flexible Latin hypercube sampling," Computers \& Geosciences, vol. 67, no. 1, pp. 62-68, 2014.

[27] D. Schiavo, L. C. Trevizan, E. R. Pereira-Filho, and J. A. Nóbrega, "Evaluation of the use of multiple lines for determination of metals in water by inductively coupled plasma optical emission spectrometry with axial viewing," 
Spectrochimica Acta Part B: Atomic Spectroscopy, vol. 64, no. 6 , pp. 544-548, 2009.

[28] C. Willmott and K. Matsuura, "Advantages of the mean absolute error (MAE) over the root mean square error (RMSE) in assessing average model performance," Climate Research, vol. 30, no. 1, pp. 79-82, 2005.

[29] N. J. D. Nagelkerke, "A note on a general definition of the coefficient of determination," Biometrika, vol. 78, no. 3, pp. 691-692, 1991.

[30] M. E. Johnson, L. M. Moore, and D. Ylvisaker, "Minimax and maximin distance designs," Journal of Statistical Planning and Inference, vol. 26, no. 2, pp. 131-148, 1990.

[31] R. Jin, W. Chen, and A. Sudjitanto, "On adaptive sampling for global metamodeling in engineering design," 2002.

[32] A. I. J. Forrester and A. J. Keane, "Recent advances in surrogate-based optimization," Progress in Aerospace Sciences, vol. 45 , no. $1-3$, pp. 50-79, 2009.

[33] L. E. Zerpa, N. V. Queipo, S. Pintos, and J.-L. Salager, "An optimization methodology of alkaline-surfactant-polymer flooding processes using field scale numerical simulation and multiple surrogates," Journal of Petroleum Science and Engineering, vol. 47, no. 3-4, pp. 197-208, 2005.

[34] A. I. J. Forrester, A. Sobester, and A. J. Keane, Engineering Design via Surrogate Modelling: A Practical Guide, DBLP, Beijing, China, 2008.

[35] Y. J. Li, J. Zhang, Y. Y. Cao et al., "Forecasting of aero-engine performance trend based on fuzzy information granulation and optimized SVM," Journal of Aeronautical Dynamics, vol. 32, no. 12, pp. 3022-3030, 2017.

[36] T. Goel, R. T. Haftka, W. Shyy, and N. V. Queipo, "Ensemble of surrogates," Structural and Multidisciplinary Optimization, vol. 33, no. 3, pp. 199-216, 2007.

[37] F. A. C. Viana, R. T. Haftka, and L. T. Watson, "Efficient global optimization algorithm assisted by multiple surrogate techniques," Journal of Global Optimization, vol. 56, no. 2, pp. 669-689, 2013. 